\title{
PAPER
}

\section{Spontaneous haemorrhage into metastatic brain tumours after stereotactic radiosurgery using a linear accelerator}

\author{
H Suzuki, S Toyoda, M Muramatsu, T Shimizu, T Kojima, W Taki
}

J Neurol Neurosurg Psychiatry 2003;74:908-912

See end of article for
authors' affiliations
$\ldots \ldots \ldots \ldots \ldots \ldots \ldots \ldots \ldots$
Correspondence to:
Dr Hidenori Suzuki,
Department of
Neurosurgery, Mie
University School of
Medicine, 2-174 Edobashi,
Tsu, Mie 514-8507, Japan;
suzuki02@
clin.medic.mie-u.ac.jp
Received 10 June 2002
In final revised form
3 February 2003
Accepted
7 February 2003

\begin{abstract}
Objective: To determine the incidence and clinical characteristics of spontaneous haemorrhage into metastatic brain tumours after radiosurgery.

Methods: Intratumour haemorrhage rate, clinical features, and treatment were evaluated in 54 patients with 131 brain metastases of varying origin who were treated using linear accelerator radiosurgery. The marginal dose was maintained constant at 20 or $25 \mathrm{~Gy}$, irrespective of tumour size.

Results: Haemorrhage was identified in $7.4 \%$ of the metastases (five tumours in four patients) before radiosurgery and in $18.5 \%$ (10 tumours in 10 patients) after radiosurgery. In three cases, haemorrhage into the tumour after radiosurgery was symptomatic. Half the haemorrhages occurred within one month of radiosurgery. The changes in tumour size observed at the time of haemorrhage were an increase in one tumour, no change in five, and a decrease in four. Haemorrhage into a fumour after radiosurgery was more likely to occur in female patients, in tumours with a larger volume on pretreatment neuroimaging, and in tumours treated with a larger number of isocentres or a higher maximum dose. Haemorrhagic features in the patients or their tumours on presurgical assessment were not disposing factors to haemorrhage after radiosurgery.

Conclusions: When larger brain metastases are aggressively treated by radiosurgery, better local control may be attained but there may also be a higher risk of haemorrhage soon after the treatment.
\end{abstract}

S ereotactic radiosurgery has been reported to be an effecive form of treatment with acceptably low rates of complications for patients with brain metastases, irrespective of tumour histology. ${ }^{1}$ For local control, quality of life, and the ratio of cost to benefit, there are strong arguments in favour of radiosurgery, especially for radioresistant metastases, despite the lack of improvement in survival duration. ${ }^{2}$ However, spontaneous haemorrhage into metastatic brain tumours after radiosurgery has not been sufficiently investigated. Haemorrhage into metastatic tumours is commonly reported ${ }^{3}$ and may cause the acute onset of either a new focal neurological deficit or worsening of pre-existing focal deficits, commonly associated with a deterioration in the level of consciousness. ${ }^{4}$ We undertook this study to determine the incidence and clinical characteristics of haemorrhage into metastatic brain tumours after radiosurgery.

\section{METHODS \\ Patient and tumour characteristics}

Between November 1994 and August 2000, 72 patients in whom a metastatic brain tumour had been diagnosed underwent linear accelerator (LINAC) radiosurgery in our unit. Eighteen of these patients, who were referred to our unit from local hospitals and attended follow up according to each hospital's protocol, were excluded from the study. The remaining 54 patients with 131 various brain metastases constituted the population of this retrospective analysis study. There were 33 men and 21 women, mean age 63 years (range 27 to 96).

Brain metastases were assessed by both non-contrast or contrast enhanced computed tomography (CT) and Tl weighted, T2 weighted, or gadolinium enhanced magnetic resonance imaging (MRI). Tumours considered for radiosurgery had a maximum diameter of $35 \mathrm{~mm}$. The number of brain metastases ranged from one to eight (mean two). Tumour volumes ranged from 0.05 to $19.8 \mathrm{~cm}^{3}$ (mean $4.4 \mathrm{~cm}^{3}$ ). Five tumours in four patients were haemorrhagic (table 1). Haemorrhage was defined as acute blood accumulation demonstrated on CT or MRI at the time of a neurological event, or as changes in signal intensity identical with a haemorrhage on serial MRI.

The primary malignancy was adenocarcinoma in 30 cases (17 lung, three stomach, two colon, two rectum, two breast, one thyroid, one ovary, one prostate, and one gallbladder), squamous cell carcinoma in 12 ( 11 lung and one breast), large cell carcinoma in four (lung), small cell carcinoma in three (lung), renal cell carcinoma in two, sarcoma in two (one lung and one synovium), and melanoma in one (table 1). Histological tumour confirmation was not obtained routinely because nearly all patients had contrast enhancing mass lesions associated with their primary tumours. Biopsies were done when imaging features were atypical or the diagnosis of primary malignancy was remote.

Twenty nine patients had disease limited to the brain and 25 had additional metastatic sites that included lung, bone, adrenal gland, liver, and lymph nodes. A patient's systemic disease was either stable or under reasonable control. Despite initial neurological deficits, all patients presented with a Karnofsky performance scale ${ }^{5}$ score of $70 \%$ or greater. Ten patients received conventional whole brain or focal radiation therapy: five before, one immediately after, and four concomitant with radiosurgical treatment. The mean total radiation dosage was 31.9 Gy (range 18 to 46 ), given using standard time-dose fractionation schemes.

\section{Follow up review}

Patients attended follow up at two weeks after radiosurgery and thereafter at intervals of one month. Patients were also seen if their condition changed. At each follow up visit all patients underwent a clinical examination and MRI. CT was done if needed. The mean follow up period was 6.4 months (range 0.5 to 60 ).

\section{Radiosurgical procedure}

A Fischer stereotactic head ring (Leibinger, Freiburg, Germany) was fixed to the patient's head under local anaesthesia. Contrast enhanced CT was done using a stereotactic localiser. 
Table 1 Primary malignancy and intratumour haemorrhage before and after radiosurgery

\begin{tabular}{|c|c|c|}
\hline \multirow[b]{2}{*}{ Primary malignancy } & \multicolumn{2}{|l|}{ Haemorrhagic tumours } \\
\hline & Pretreatment haemorrhagic & Post-treatment haemorrhagic \\
\hline $\begin{array}{l}\text { Adenocarcinoma }(\mathrm{n}=30) \\
\text { (17 lung, } 3 \text { stomach, } 2 \text { rectum, } 2 \text { breast, } 2 \text { colon, } 1 \text { thyroid, } 1 \text { ovary, } 1 \\
\text { prostate, } 1 \text { gallbladder) }\end{array}$ & $\begin{array}{l}2 \text { patients } \\
\text { (1 lung, } 1 \text { stomach) }\end{array}$ & $\begin{array}{l}5 \text { patients } \\
\text { ( } 3 \text { lung, } 1 \text { stomach, } 1 \text { rectum (S) }\end{array}$ \\
\hline $\begin{array}{l}\text { Squamous cell carcinoma }(n=12) \\
\text { (11 lung, } 1 \text { breast) }\end{array}$ & 0 & 1 \\
\hline $\begin{array}{l}\text { Sarcoma ( } \mathrm{n}=2 \text { ) } \\
\text { (1 lung, } 1 \text { synovium) }\end{array}$ & 1 synovium & 1 lung (S) \\
\hline $\begin{array}{l}\text { Others } \\
\text { Large cell carcinoma (lung) ( } n=4) \\
\text { Small cell carcinoma (lung) }(n=3) \\
\text { Renal cell carcinoma ( } n=2) \\
\text { Melanoma }(n=1)\end{array}$ & $\begin{array}{l}1 \\
0 \\
0 \\
0\end{array}$ & $\begin{array}{l}1 \\
1 \\
0 \\
1(S)\end{array}$ \\
\hline
\end{tabular}

The patient's entire head was scanned using $2 \mathrm{~mm}$ contiguous slices. For image analysis, Fischer STP3 treatment planning equipment for stereotactic radiosurgery (Leibinger, Freiburg, Germany), was employed. The radiation source was a standard LINAC (EXL-15SP; Mitsubishi, Amagasaki, Japan) with nine additional collimators ( 5 to $23 \mathrm{~mm}$ ).

Eighty seven lesions were treated using a single isocentre ( 1 to 16 noncoplanar arcs). Different combinations of the arc span, arc weighting, angle of arc incidence, and number of arcs were used to achieve maximum conformity of treatment dose at the borders of the tumour. Forty four lesions with irregular shapes were treated using two (29 lesions), three (nine lesions), four (five lesions), or five isocentres (one lesion).

During the first three years of the study, a dose of 20 Gy was delivered at the tumour margin irrespective of tumour size. Thereafter, the marginal dose prescribed was increased to 25 Gy irrespective of tumour size; this was intended to attain a better local response or control rate, but it was unsuccessful in this, at least within the follow up period in this study. As a result, larger lesions received a higher maximum dose. Patients were usually discharged on the morning after treatment, receiving steroids and anticonvulsants.

\section{Statistical analysis}

All values are expressed as means (SD). Comparisons between the two groups were made using unpaired $t$ tests, $\chi^{2}$ tests, or Fisher's exact test, as appropriate. Significance was assigned when the probability $(\mathrm{p})$ value was less than 0.05 .

\section{RESULTS}

\section{Radiological response of tumours after radiosurgery}

Of 131 tumours in 54 patients, 31 (23.7\%) disappeared, 61 $(46.6 \%)$ decreased in size, and $31(23.7 \%)$ became stable in size during the period of follow up. Local control was achieved in 123 tumours $(93.9 \%)$ in 52 patients $(96.3 \%)$. The local control rate was not significantly affected by the patients' age or sex, the tumour histology, tumour volume, the number of lesions or isocentres, the marginal dose, or any association with conventional radiotherapy. Local tumour progression was observed in eight tumours $(6.1 \%)$ in two patients $(3.7 \%)$. In all, 19 patients developed new metastases outside the field of radiosurgery; of these, six received conventional whole brain radiation treatment and four underwent repeat radiosurgery. Imaging follow up in seven of the 10 tumours treated with repeat radiosurgery showed disappearance of the tumour or decreased tumour size at 6.5 to 11.5 months after the procedure. One patient with three tumours was lost to follow up.
Transient complications after radiosurgery were observed in nine patients, who experienced perioperative nausea, vomiting, or dizziness. In one of these patients, nausea and vomiting were caused by haemorrhage into a tumour. One patient developed delayed radiation injury (radiation necrosis) within the follow up period, which required surgical resection. Nine patients had delayed intratumour haemorrhage. All haemorrhages were in treated tumours, and none was observed in a newly developed intraparenchymal metastasis outside the field of radiosurgery. Three additional patients developed local tumour recurrence; one required surgery and two underwent a second radiosurgery. The marginal doses in the second radiosurgery were $12 \mathrm{~Gy}$ in two tumours and $20 \mathrm{~Gy}$ in one. Imaging follow up in three tumours treated with repeat radiosurgery showed stable or decreased tumours at 3.0 to 11.5 months after the second procedure.

\section{Intratumour haemorrhage after radiosurgery}

Intratumour haemorrhages after radiosurgery were observed in 10 tumours in 10 patients ( $18.5 \%)$, as described above. No patient had coagulopathy. These haemorrhages occurred in five of 30 adenocarcinomas, one of 12 squamous cell carcinomas, one of four large cell carcinomas, one of three small cell carcinomas, one of two sarcomas, and in the sole melanoma (table 1). The interval between radiosurgery and haemorrhage was one day in one tumour, two weeks in two, one month in two, two months in two, three months in one, and four months in two. The haemorrhages were symptomatic in three of the 10 patients; two (one with a rectal carcinoma and the other with a melanoma) recovered with conservative treatment; the remaining patient (with an alveolar sarcoma) died of brain herniation following rebleeding nine months after radiosurgery (five months after the initial bleed). In relation to pretreatment size, at the time of the haemorrhage tumour size increased in one tumour, remained unchanged in five, and decreased in four. Nine patients received no additional treatment: one tumour disappeared, four decreased in size, three remained stable in size, and one increased in size, with rebleeding and brain herniation at the final follow up. One patient who had an asymptomatic intratumour haemorrhage with no change in tumour size underwent stereotactic aspiration of a haematoma in the tumour, and then had repeat radiosurgery with a marginal dose of $15 \mathrm{~Gy}$ (at the attending physician's preference); this tumour (a lung adenocarcinoma) remained unchanged in size but did not rebleed until the last follow up.

Although radiosurgery attained good local control independent of any haemorrhagic manifestations, only three 
Table 2 Clinical characteristics of 54 patients with or without intratumour haemorrhage after radiosurgery

\begin{tabular}{lll}
\hline & Post-treatment haemorrhagic & Post-treatment non-haemorrhagic \\
\cline { 1 - 2 } No of patients & 10 (symptomatic in 3) & 44 \\
Age (years) & $58(14)$ & $65(13)$ \\
Sex ratio (male/female) & $3 / 7^{*}$ & $30 / 14$ \\
Mean follow up period (months) & $6.4(3.5)$ & $6.3(9.2)$ \\
Mean No of lesions & $1.8(1.5)$ & $2.2(1.6)$ \\
Rate of multiple lesions (\%) & 30.0 & 50.0 \\
Additional irradiation (Number of patients (\%)) & $2(20.0)$ & $8(18.2)$ \\
Pretreatment haemorrhage (Number of patients (\%)) & $2(20.0)$ & $2(4.5)$ \\
\hline & & \\
Values are mean (SD) unless stated. & & \\
*There were significantly more women in the post-treatment haemorrhagic group than in the post-treatment non-haemorrhagic group (p < 0.05). \\
Post-treatment haemorrhagic/non-haemorrhagic, patients with or without intratumour haemorrhage after radiosurgery; pretreatment haemorrhage, patients
\end{tabular}

Table 3 Clinical characteristics in 131 tumours with or without intratumour haemorrhage after radiosurgery

\begin{tabular}{lll}
\hline & Post-treatment haemorrhagic & Post-treatment non-haemorrhagic \\
\hline No of lesions & 10 (symptomatic in 3) & 121 \\
Mean volume $\left(\mathrm{cm}^{3}\right)$ & $9.2(5.3) \dagger$ & $4.0(5.1)$ \\
Mean No of isocentres & $2.3(1.3) \dagger$ & $1.5(0.8)$ \\
Mean marginal dose (Gy) & $20.2(5.4)$ & $20.8(4.0)$ \\
Mean maximum dose (Gy) & $40.5(20.3)^{*}$ & $29.8(6.3)$ \\
Local response rate (\%) & 50 & 73.5 \\
Local control rate (\%) & 90 & 95.6 \\
Pre-treatment haemorrhage (No of lesions (\%)) & 0 & $5(4.1)$ \\
\hline
\end{tabular}

Values are mean (SD) unless stated.

${ }^{*} p<0.05, \dagger p<0.005$ : significantly different between post-treatment haemorrhagic and post-treatment non-haemorrhagic tumours.

Post-treatment haemorrhagic/non-haemorrhagic, tumours with or without intratumoural haemorrhage after radiosurgery; pretreatment haemorrhage, tumours with intratumour haemorrhage before radiosurgery.

patients were alive at the last follow up: two of these retained their pretreatment quality of life but the other was severely disabled owing to a spinal metastasis. The cause of death was systemic malignancies in five patients (primary tumours in three and secondary tumours in two), brain tumour haemorrhage in one, and myocardial infarction in one. Thus, other than in the one patient who died as a direct result of brain tumour haemorrhage, intratumour haemorrhages after radiosurgery appeared to have no influence on long term outcome.

Among patient characteristics, the only significant factor that differentiated haemorrhagic tumours from nonhaemorrhagic tumours after radiosurgery was being female (table 2): haemorrhages were observed in three of the 33 men $(9.1 \%)$ and seven of the 21 women $(33.3 \%)(p<0.05)$. In relation to treatment variables, the mean tumour volume in haemorrhagic tumours was significantly greater than in nonhaemorrhagic tumours $(\mathrm{p}<0.005$; table 3$)$. The mean number of isocentres and maximum doses given were also significantly greater in the haemorrhagic tumours than in the non-haemorrhagic tumours $(\mathrm{p}<0.05)$; this was attributed to the difference in tumour volume and the radiosurgery strategy.

\section{Patients with haemorrhagic tumours before radiosurgery}

Four patients $(7.4 \%)$ had a total of five haemorrhagic tumours before radiosurgery (table 1). Two of these patients each had one intratumour haemorrhage after radiosurgery, which occurred two weeks and two months after the treatment, respectively. The primary malignancies were a lung adenocarcinoma and a lung large cell carcinoma. Both patients were female. They developed new intratumour haemorrhages into treated tumours in which no haemorrhage was evident before the radiosurgery. No rebleeding into tumours that were haemorrhagic before treatment was observed after radiosurgery. The tumour volume of each newly haemorrhagic tumour increased. Of the 50 patients with no haemorrhage before radiosurgery, eight had a total of eight intratumour haemorrhages after radiosurgery. The rate of haemorrhage after radiosurgery was not significantly different between the patients with and without pretreatment haemorrhages.

The mean tumour volume in haemorrhagic tumours was significantly greater than in non-haemorrhagic tumours both before and after radiosurgery $(p<0.01$ and $p<0.005$, respectively). However, in neither haemorrhagic nor nonhaemorrhagic tumours did the mean tumour volume differ significantly before and after the radiosurgery (10.6 (6.1) $v 9.2$ (5.3), and $4.2(5.1) v 4.0(5.1) \mathrm{cm}^{3}$, respectively). Local response and control rates were good whether or not intratumour haemorrhage occurred, either before or after radiosurgery.

\section{DISCUSSION}

The effect of radiosurgery on spontaneous haemorrhage into metastatic brain tumours has not been sufficiently investigated. This study showed that haemorrhage was observed in $7.4 \%$ of patients (3.8\% of tumours) before radiosurgery and in $18.5 \%$ of patients $(7.6 \%$ of tumours) after radiosurgery. This would not necessarily imply that radiosurgery increases the likelihood of haemorrhage, as the incidence of intratumour haemorrhage is quite different between the early and later stages of brain metastases, which grow very rapidly. Rather, the incidence of haemorrhage after radiosurgery (18.5\%) was no greater than that of untreated tumours reported previously $(0-50 \%) .^{3}$ However, it is not possible to state definitively whether radiosurgery increases, does not change, or decreases the risk of haemorrhage, because of the lack of a control group. However, $50 \%$ of the observed haemorrhages occurred within one month of the procedure. As most haemorrhagic tumours were under good local control at the time of the haemorrhage and thereafter, it is likely that radiosurgery may have contributed to the haemorrhages which occurred at relatively short intervals after the treatment.

The only significant complication of stereotactic radiosurgery has been delayed radiation injury (radiation necrosis), 
which typically occurs three months or more after treatment. ${ }^{6}$ The immediate complications occasionally seen with craniotomy, such as haemorrhage and infection, do not occur after radiosurgery. However, there have been several recent reports of intratumour haemorrhage after radiosurgery in patients with brain metastases, ${ }^{1-10}$ although radiosurgery did not appear to increase the incidence of haemorrhage. ${ }^{19}{ }^{11}$ A review of published reports revealed that the rate of haemorrhagic complications was between $1 \%$ and $8 \%$, while the radiation complication rate was $0 \%$ to $7 \%$, although the definition of a haemorrhage or a radiation necrosis was not uniform. ${ }^{2}{ }^{12}$ Mori et al reported that intratumour haemorrhage occurred in $10 \%$ of patients with melanoma metastases before radiosurgery, and in $6 \%$ (three of 42 patients) at the radiosurgery site. ${ }^{1}$ Suzuki et al and Kim et al reported that one of 24 patients and one of 53 patients, respectively, with various brain metastases, died from tumour haemorrhage after radiosurgery. ${ }^{70}$ The interval between radiosurgery and the intratumour haemorrhage was two days ${ }^{10}$ and three days ${ }^{7}$ in lung cancer, one month in melanoma, ${ }^{13}$ six weeks in breast cancer, ${ }^{8}$ and between one and seven months in melanoma. ${ }^{1}$ Intratumoral haemorrhage was observed in tumours with a large volume in pretreatment neuroimaging ${ }^{8}{ }^{9}$ or with increased tumour size despite radiosurgery. ${ }^{1}$ Most of the reported patients required craniotomy $^{813}$ or died because of tumour haemorrhage. ${ }^{710}$ These findings are consistent with the present observations, even though our study included more asymptomatic cases, resulting in a higher haemorrhage rate after radiosurgery.

The biological effect of radiosurgery is the early direct injury to tumour cells followed by late injury to the tumour vasculature within the high dose target volume. ${ }^{14}{ }^{15}$ In brain metastases, radiosurgery appears primarily to affect the neoplastic cells rather than the tumour vasculature, causing acute necrosis of the rapidly proliferating malignant cells. ${ }^{14}$ The endothelial cells, when injured following irradiation, can be involved in the release of a variety of cytokines which may produce thrombotic lesions or disrupt the blood-brain barrier. ${ }^{16}$ A persistent though variable disruption of the bloodbrain barrier was demonstrated from two to 24 weeks postirradiation, with an initial peak at six weeks, leading to oedema and haemorrhage. ${ }^{16}$ On the other hand, ongoing vessel wall thickening after radiosurgery may decrease the risk of haemorrhage, although it is possible that an increase in intravascular outflow resistance owing to radiation induced venous obliteration could promote haemorrhage. ${ }^{9}$ Motozaki et al suggested that radiosurgery for large, vessel-rich tumours caused necrosis in the tumour and blood flow congestion in residual neoplastic tissue, leading to haemorrhage. ${ }^{8}$ Park et al proposed that a metastatic brain tumour with pre-existing haemorrhage was predisposed to bleeding of unknown aetiology, irrespective of whether or not there was radiosurgery. ${ }^{9}$ In the present series, variables associated with tumour haemorrhage after radiosurgery were female sex, larger tumours, a larger number of isocentres, and higher maximum dose. In the present radiosurgical strategy, larger tumours were treated with a larger number of isocentres and a higher maximum dose. Moreover, the mean tumour volume in haemorrhagic tumours was not significantly different either before or after radiosurgery. Considering that larger size of untreated tumours may be associated with a greater incidence of haemorrhage, ${ }^{17}{ }^{18}$ one possibility is that haemorrhagic complications may merely express the natural history of large tumours. ${ }^{11}$ This possibility cannot be excluded because our study lacked a control group and no data defining a relation between haemorrhagic risk and tumour size have been reported. However, an unusual finding in the present study was that $50 \%$ of the observed haemorrhages occurred within one month of the procedure, irrespective of whether tumours were under good local control. Another more plausible possibility is that brain metastases may be predisposed to haemorrhage at relatively short intervals after radiosurgery, because radiosurgery causes acute necrosis of tumour cells before the obliteration of the tumour vasculature. ${ }^{14}$ Although large tumours with pre-existing intratumour haemorrhage did not bleed after radiosurgery, this may be because the tumour tissue within the higher dose target volume had been replaced by haematoma before the treatment. The reason why women were at higher risk of bleeding after radiosurgery is unclear, and this finding is also inconsistent with the findings relating to untreated tumours. ${ }^{17}$

The risk for the development of complications from radiosurgery has been linked with several clinical, biological, and treatment planning variables such as target dose inhomogeneity, maximum target dose, maximum normal tissue dose, number of treatment isocentres, and target volume. ${ }^{11}{ }^{19}$ On the other hand, variables most significantly associated with better local control were small tumour size, higher treatment dose, and radiosurgical treatment on presentation rather than after recurrence. ${ }^{15}$ Thus a smaller volume was associated with better control $^{7}$ while a larger volume increased the risk of complications. ${ }^{13}$ In the present study, radiosurgery attained good local control for any size of brain metastasis, probably because the marginal dose prescribed was constant at 20 or 25 Gy, irrespective of tumour size. However, the incidence of post-radiosurgery haemorrhage in the present study was relatively increased (at $18.5 \%$ ) compared with previous studies ( $1-8 \%)$, although the definition of a haemorrhage was not uniform. ${ }^{2}{ }^{12}$ The explanation may lie in the radiosurgical technique used, whereby in order to keep the marginal dose constant, a significant increase in the maximum dose in the larger tumours was allowed. Owing to the relative difficulty of using multiple isocentres in an LINAC based system compared with an alternative common radiosurgical technique, gamma knife radiosurgery, ${ }^{20}$ multiple isocentre treatments were more closely related to tumour size than plan conformity, resulting in higher tumour dose inhomogeneity and therefore a higher maximum dose in larger tumours. A higher treatment dose may promote acute tumour necrosis before the obliteration of the tumour vasculature, leading to a higher rate of haemorrhagic complications. If so, radiosurgical treatment planning alternatives to high maximum dose may decrease the haemorrhagic complications. In this study, larger tumours were typically treated with 20 to 25 Gy prescribed at the $40-50 \%$ isodose (therefore a maximum dose of 50 to $60 \mathrm{~Gy}$ ) using two to three isocentres and conventional circular collimators. Whether the haemorrhagic complications can be reduced by reductions in maximum dose remains an important area for further investigation. For larger tumours, additional treatment options such as surgery may also have to be considered.

\section{...............}

\section{Authors' affiliations}

H Suzuki, M Muramatsu, T Shimizu, T Kojima, Department of Neurosurgery, Mie Prefectural General Medical Centre, Mie, Japan S Toyoda, Department of Radiology, Mie Prefectural General Medical Centre

W Taki, Department of Neurosurgery, Mie University School of Medicine, Mie, Japan

Competing interests: none declared

\section{REFERENCES}

1 Mori Y, Kondziolka D, Flickinger JC, et al. Stereotactic radiosurgery for cerebral metastatic melanoma. Factors affecting local disease control and survival. Int J Radiat Oncol Biol Phys 1998;42:581-9.

2 Nataf F. Radiosurgery for brain metastases. Neurochirurgie 1999;45:393-7. [[In French; English abstract.]]

3 Kondziolka D, Bernstein M, Resch L, et al. Significance of hemorrhage into brain tumors. Clinicopathological study. J Neurosurg 1987;67:852-7.

4 Kase CS. Intracerebral hemorrhage. Non-hypertensive causes. Stroke 1986;17:590-5.

5 Karnofsky DA, Burchenal JH. The clinical evaluation of chemotherapeutic agents in cancer. In: MacLeod CM, ed. Evaluation of chemotherapeutic agents. New York: Columbia University Press, 1949:191-205. 
6 Chin LS, Ma L, DiBiase S. Radiation necrosis following gamma knife surgery. A case-controlled comparison of treatment parameters and long-term clinical follow up. J Neurosurg 2001;94:899-904.

7 Kim DG, Chung H-T, Gwak H-S, et al. Gamma knife radiosurgery for brain metastases. Prognostic factors for survival and local control. J Neurosurg 2000;93(suppl 3):23-9.

8 Motozaki T, Ban S, Yamamoto T, et al. Peritumoral hemorrhage after radiosurgery for metastatic brain tumor. A case report. No Shinkei Geka 1994;22:789-93. [[In Japanese; English abstract.]]

9 Park C-K, Kim DG, Gwak H-S, et al. Intracerebral hemorrhage after $\gamma$-knife surgery for metastatic brain tumor. J Radiosurg 2000;3:17-20.

10 Suzuki S, Omagari J, Nishio S, et al. Gamma knife radiosurgery for simultaneous multiple metastatic brain tumors. J Neurosurg 2000;93(suppl 3):30-1.

11 Nedzi LA, Kooy H, Alexander E, et al. Variables associated with the development of complications from radiosurgery of intracranial tumors. Int J Radiat Oncol Biol Phys 1991;21:591-9.

12 Flickinger JC, Lunsford LD, Somaza S, et al. Its role in brain metastasis management. Neurosurg Clin N Am 1996;7:497-504.

13 Somaza S, Kondziolka D, Lunsford LD, et al. Stereotactic radiosurgery for cerebral metastatic melanoma. J Neurosurg 1993;79:661-6.
14 Abdelaziz OS. Radiosurgery in neurosurgical practice IV. Stereotactic radiosurgery for metastatic brain disease. Neurosurg $Q$ 2000;10:131-5.

15 Friedman WA, Foote KD. Linear accelerator radiosurgery in the management of brain tumours. Ann Med 2000;32:64-80.

16 Rubin P, Gash DM, Hansen JT, et al. Disruption of the blood-brain barrier as the primary effect of CNS irradiation. Radiother Oncol 1994;31:51-60

17 Wakai S, Yamakawa K, Manaka S, et al. Spontaneous intracranial hemorrhage caused by brain tumor. Its incidence and clinical significance. Neurosurgery 1982;10:437-44

18 Zimmerman RA, Bilaniuk LT. Computed tomography of acute intratumoral hemorrhage. Radiology 1980;135:355-9.

19 Abdelaziz OS. An overview of radiosurgery applications in neurosurgery. Part 2. Brain tolerance to radiosurgery. Correlation with predictable complications. Neurosurg Q 1999;9:251-7.

20 Abdelaziz OS. An overview of radiosurgery applications in neurosurgery. Part 1. State-of-the-art radiosurgery. Neurosurg $Q$ $1999 ; 9: 241-50$

\section{NEUROLOGICAL PICTURE}

\section{Median nerve neurofibroma}

A 61 year old man presented with a 6 months history of intermittent paraesthesia and numbness in the first three radial digits of the right hand. Initially, the symptoms occurred during his activity as masseur when performing vigorous movements of the elbow. Later, a pea sized mass could be palpated at the distal medial upper arm. The electrophysiological examination was normal. High resolution ultrasonography demonstrated a $2 \mathrm{~cm}$ by $1 \mathrm{~cm}$ ovoid structure (fig l, white arrow) in the continuity of the median nerve (arrowheads). On operation a soft mass growing diffusely among the median nerve fascicles could be microscopically excised (fig 2). The tumour was identified as a neurofibroma on pathological examination. Postoperatively the patient experienced a full relief of symptoms with normal motor and sensory function of the median nerve.

$H$ Kele, R Verheggen

Department of Neurosurgery, Georg August University, Robert Koch Strasse 40, 37075 Goettingen, Germany

Correspondence to: Dr H Kele; hkele@gwdg.de
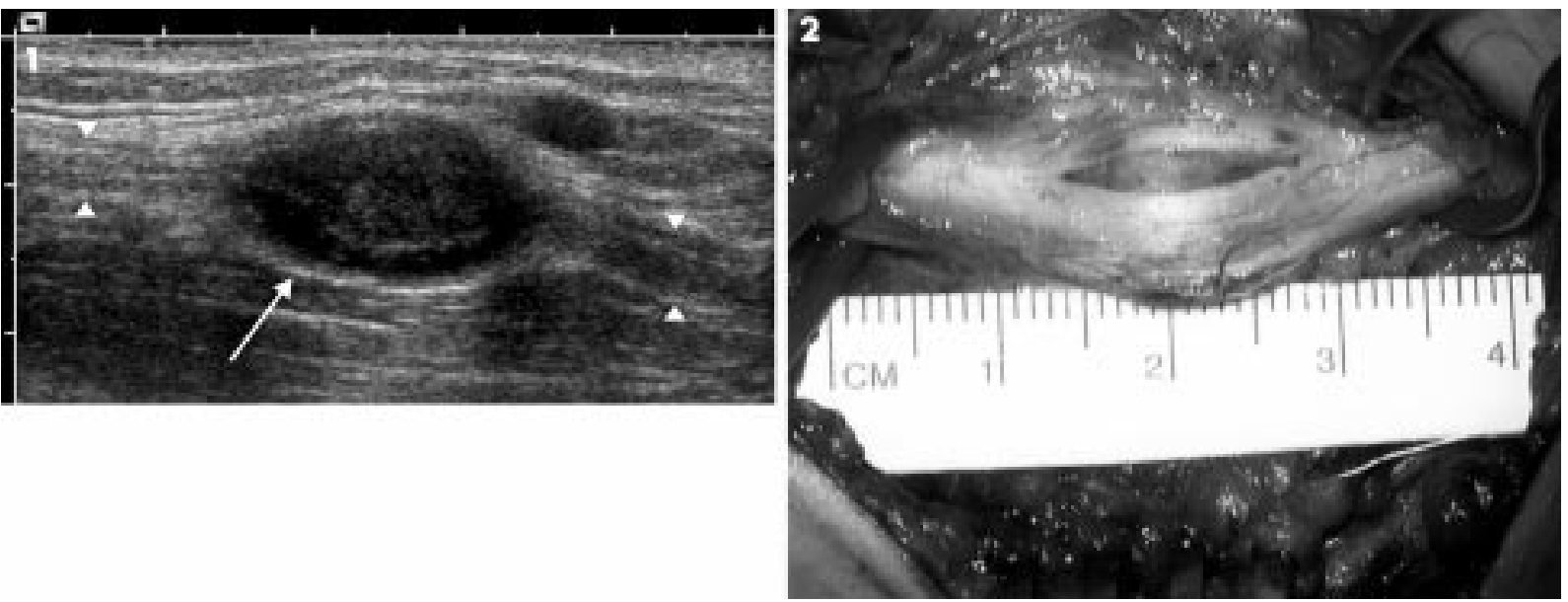